\title{
APPLICATIONS OF FTIR SPECTROSCOPY IN ENVIRONMENTAL STUDIES SUPPORTED BY TWO DIMENSIONAL CORRELATION ANALYSIS
}

\author{
M. MECOZZI* \\ F. MOSCATO \\ M. PIETROLETTI \\ F. QUARTO \\ F. OTERI \\ A.M. CICERO
}

Received: 05/10/07

Accepted: 10/12/07
Istituto Centrale per la Ricerca Scientifica

e Tecnologica Applicata al Mare (ICRAM)

via di Casalotti 300, Rome, Italy

\section{ABSTRACT}

In this paper, we report the joint use of FTIR spectroscopy and two dimensional correlation analysis (2DCORR) applied to the identification of the aggregation pathways of natural organic matter (i.e. humic substance) extracted from marine sediments and in the second case of study, to the comparison of the molecular modifications caused by the actions of different pollutants on a marine algae used as biomarker of environmental quality. In the first application, the extractable humic substance (HS) from sediments was ultra-filtered to obtain seven sub-fractions of HS with molecular weight $(\mathrm{mw})<1 \mathrm{kDa}$ and $>100 \mathrm{kDa}$. After the examination of FTIR spectra of each sediment samples, 2DCORR was applied to examine the molecular mechanism of the aggregation process. This approach allows to differentiate the role played by carbohydrates, lipids and proteins during the evolution of the aggregation process. Carbohydrates are significant in any phase of the aggregation while proteins though significant in any phase of the aggregation seem to play a role strictly related to polymerisation reaction with carbohydrates. Lipids play a completely different role in the aggregation process with respect to carbohydrates and proteins because they are significant only in sub-fractions of HS with $m w<1 \mathrm{kDa}$ and $\mathrm{mw}>5 \mathrm{kDa}$, so suggesting that their role is the so called cross-linking action (i.e. bridge among lower mw aggregate), already observed in the aggregation of terrestrial HS. In the second application of FTIR spectroscopy and 2DCORR, the acetone extracts of Dunaliella tertiolecta prepared according to the ISO standard protocol for toxicity test, were examined in presence of several inorganic and organic pollutants to study the molecular modifications caused by pollutant actions in algal cells. This approach allows to differentiate the molecular mechanisms of toxic action for the observed pollutants. Heavy metals such as $\mathrm{Hg}, \mathrm{Zn}, \mathrm{Cr}(\mathrm{VI})$ and $\mathrm{Cu}$ cause modifications of carbohydrate lipid and nucleic acid contents and structural modifications (i.e. inter and intra molecular hydrogen bonds) of these biomolecules simultaneously. Glycols compounds cause modifications of carbohydrates lipids and nucleic acids contents and structural modifications too as observed for heavy metals but without an evident simultaneity between the two types of modification. At last, surfactants cause modifications of carbohydrates lipids and nucleic acid contents only. These experimental results suggest that the use of FTIR spectroscopy with 2DCORR analysis is a potential tool for evaluating toxic effects in the study of environmental quality of marine samples.

KEYWORDS: FTIR spectroscopy, 2D correlation analysis, humic substance aggregation, marine sediments, ecotoxicology, pollutants, mechanisms of toxicity action. 


\section{INTRODUCTION}

Fourier Transform Infrared (FTIR) spectroscopy allows the analysis of a relevant amount of compositional and structural information concerning environmental samples (Kögel-Knaber, 2000) and recently, its joint use with 2DCORR has improved the performance of this technique in the studies of complex environmental systems (Noda and Ozaki, 2005). In fact, 2D correlation analysis is a method for visualizing the dynamic relationships among the variables in multivariate data set by applying the complex cross-correlation function. This approach allows to identify the spectral features which change in phase (i.e. linearly correlated among them) and out of phase (partially or not at all correlated among them). In more details, when we examine a complex environmental system during its dynamic (i.e. temporal or spatial) evolution by FITR spectra and in addition, we use 2DCORR to reexamine them, we can characterize the system clarifying specific aspects of the molecular mechanisms involved in the dynamic evolution of the environmental system to be studied. The study of the relationships among variables is the field of the wavelength-wavelength (WW) 2DCORR while the study of the relationships among samples is the field of the samplesample (SS) 2DCORR (Šašic et al., 2001).

In this paper, we report the joint use of FTIR spectroscopy and 2DCORR applied to two specific environmental studies. The first case of study is related to the identification of the aggregation pathways of HS samples extracted from marine sediments; the second case of study is related to the comparison of the molecular modifications caused by the actions of different pollutants on the marine algae Dunaliella tertiolecta used as biomarker of environmental quality.

\section{METHODS AND MATERIALS}

\subsection{Samples of natural organic matter from marine sediments}

The humic substance was extracted from some sediment samples of Tyrrhenian Sea and separated by precipitation at $\mathrm{pH}=2$ in the two fractions of fulvic acids (FAs) and humic acids (HAs). FAs were further fractionated in seven sub-samples of different molecular weight (mw) by means of seven different ultrafiltration membranes operating in the range between $\mathrm{mw}<1$ kDa and mw > 100kDa (Mecozzi and Pietrantonio, 2006).

\subsection{Samples of pigments extracted by an algal culture submitted to pollutants action} Cultures of Dunaliella tertiolecta, a widespread algae used for toxicity estimation of marine samples, were treated according to the ISO protocol in presence of several pollutants to obtain the extracts of their pigments (EPA Method 600/4-90-027, 1991).

\subsection{Collection of FITR spectra}

FTIR spectra were collected in diffuse reflectance (DRIFT) mode in the range between 4000 and $400 \mathrm{~cm}^{-1}$ for the study of EHS and in attenuated total reflectance (ATR) mode in the range between 4000 and $650 \mathrm{~cm}^{-1}$ for the study of algal cultures. All the spectra were submitted to a nine point smoothing filter and then saved as ASCII files. For conventional FTIR studies, spectra were normalised using the amide I band of proteins at $1650 \mathrm{~cm}^{-1}$; for 2DCORR analysis, FTIR spectra were previously autoscaled.

\subsection{D correlation analysis of FTIR spectra}

2DCORR in WW and SS mode was performed on the ASCII files of FTIR spectra by means of an in house software written in MATLAB (5.2 version) code, according to the related scientific literature (Šašic et al., 2001).

\section{Results and discussion}

\subsection{FTIR spectroscopy and 2D correlation analysis in the study of the aggregation mechanisms of HS}

FTIR spectroscopy of ultra-filtered sub-fractions of HS samples evidences relevant changes of lipid concentrations with respect to carbohydrates and proteins during the evolution of the aggregation process (Figure 1). In fact, lipids are significant in aggregates with mw lower than $1 \mathrm{kDa}$, almost negligible in the fractions with mw higher than $1 \mathrm{kDa}$ and lower than $5 \mathrm{kDa}$ and significant again in the fractions with mw higher than $5 \mathrm{kDa}$. Carbohydrates and proteins show 
different characteristics because they are significant components of the sub-fractions of organic matter. This result could be likely depend on the cross-linking (i.e. micelle formation) role played by lipids during the aggregation process of HS. The cross-linking role is different from the role played by carbohydrates and proteins, based instead on their self aggregation and polymerisations characteristics and on their specific interactions with the common metal elements of marine environment such as $\mathrm{Ca}$ and $\mathrm{Mg}$ ions.

2DCORR supports the results obtained by FTIR spectroscopy and in addition, allows some specific insights in the structure of these aggregates of organic matter. 2DCORR WW spectroscopy revealed that carbohydrates and proteins have relevant contributions to all the steps of the aggregation process (Figure 2) also by means of specific interactions among them. These interactions between carbohydrates and proteins in the formation of EHS aggregates can vary among different sediment samples but are in any case always present as shown by the autopeaks at 3400,1650 and $1150 \mathrm{~cm}^{-1}$ (Figure 2, right plot), the typical peaks of these compounds. The crosspeaks at 3400 vs 1650 , and 3400 vs $1150 \mathrm{~cm}^{-1}$ are the evidence of the interactions (polar and condensation reactions) between these biomolecules.

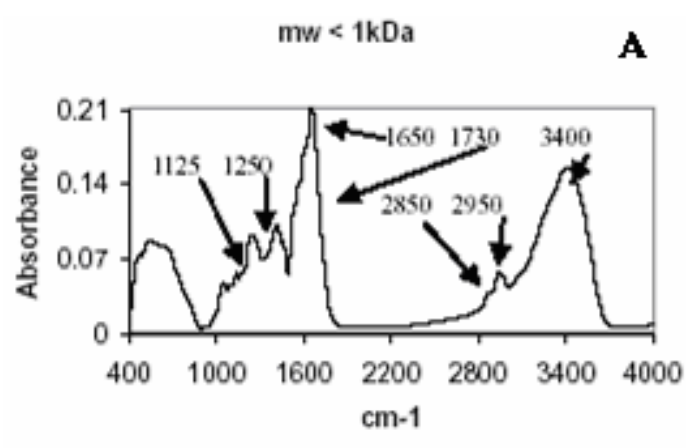

$3 \mathrm{kDa}<\mathrm{mw}<5 \mathrm{kDa}$

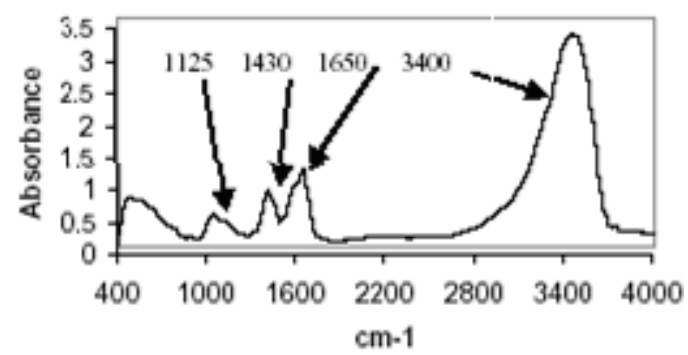

$1 \mathrm{kDa}<\mathrm{mw}<3 \mathrm{kDa}$

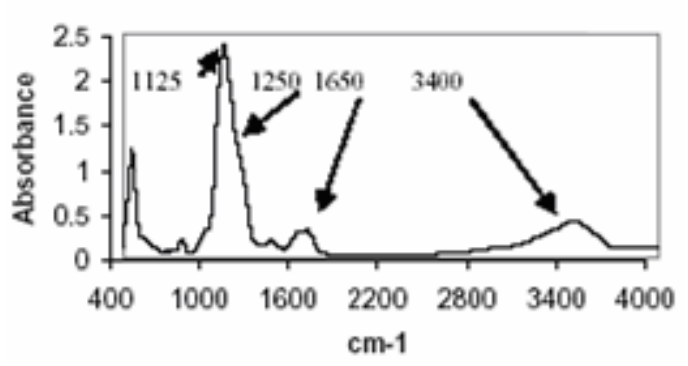

$5 \mathrm{kDa}<\mathrm{mw}<10 \mathrm{kDa}$

D

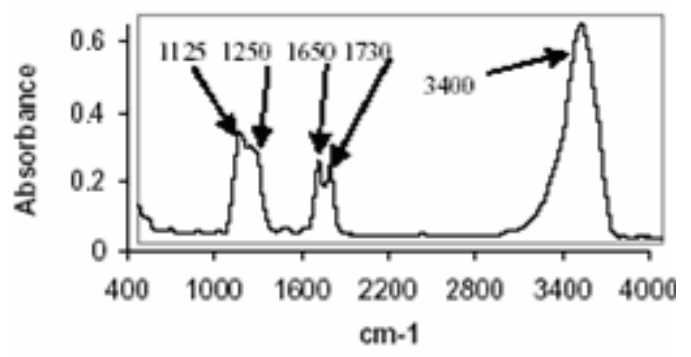

Figure 1. FTIR spectra of some sub-fractions of HS extracted from a sample sediment showing the absence of ester fatty acids (band at $1730 \mathrm{~cm}^{-1}$ ) in aggregates with mw ranging between 1 and $5 \mathrm{kDa}$ (spectra $\mathrm{B}$ and $\mathrm{C}$ )

Vice versa, lipid compounds such as fatty acids and ester fatty acids, did not show interactions with carbohydrates and proteins because their presence in the different HS fractions is never related to carbohydrate and protein contents present in samples. This finding supports the hypothesis of the cross linking role played by lipids during the aggregation process, already evidenced by conventional FTIR spectroscopy (Figure 1) (Mecozzi and Pietrantonio, 2006).

Crosspeaks of asynchronous spectra (Figure 3) at 3400 vs 1150,3400 vs 1150,3400 vs 2850, 1650 vs $1150 \mathrm{~cm}^{-1}$ show the complexity of the chemical interactions among carbohydrates, lipids and proteins which happen with an out of phase component. In addition, it should be noted that in the left 2DCORR spectra of Figure 3 there is the unique crosspeaks at 3400 vs $2850 \mathrm{~cm}^{-1}$ (i.e. the dashed arrow) related to the carbohydrate lipid interaction. This is the confirmation of the different role played by this class of biomolecules in the aggregation process of HS which is differentiated with respect to carbohydrates and proteins. 2DCORR SS spectroscopy (Figure 4) confirmed the general evidences obtained by 2D WW spectroscopy and moreover, gives the evidence that the formation of EHS aggregates is in 
any case a complex process where aggregation processes are in chemical equilibrium with degradation processes. These results agree with those of other studies concerning the aggregation mechanisms of HS (Ishiwatari, 1992; Verdugo et al., 2004).
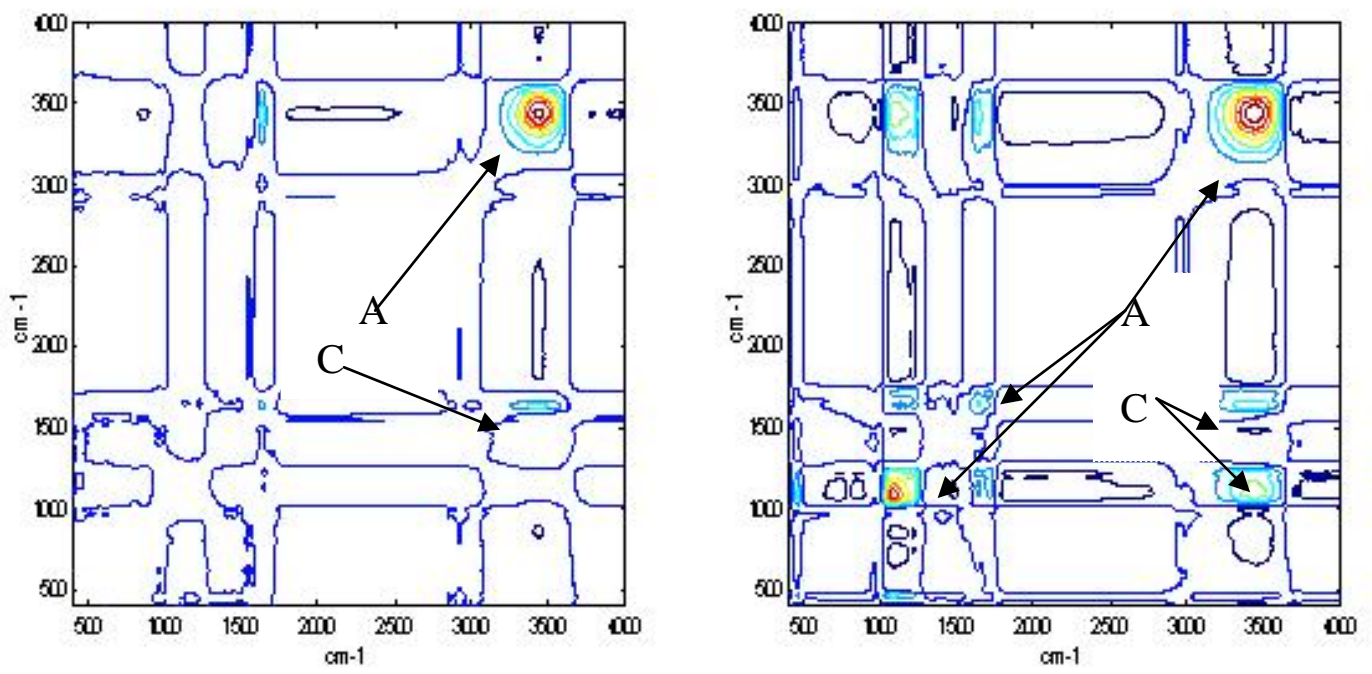

Figure 2. 2DCORR synchronous spectra as contour plot of two HS samples. "A" and "C" show autopeaks and crosspeaks respectively
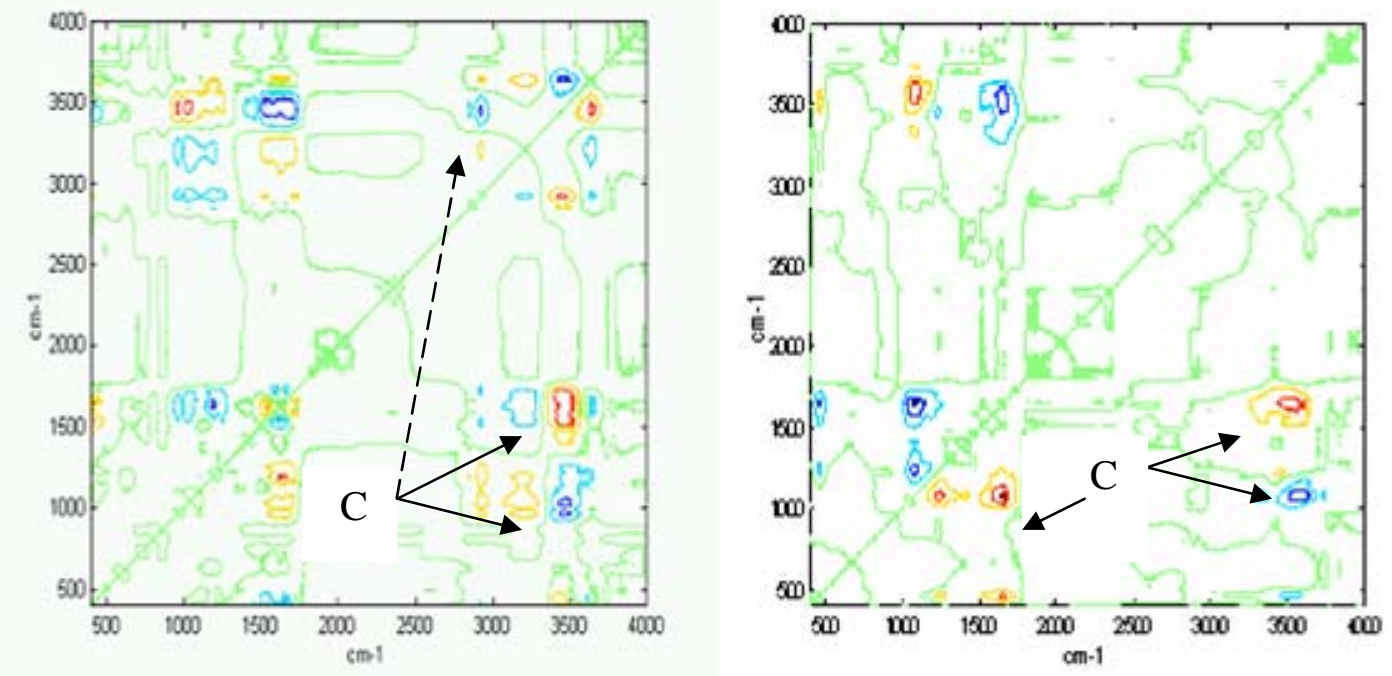

Figure 3. 2D CORR asynchronous spectra of the same HS samples of Figure 2. "C" means crosspeaks
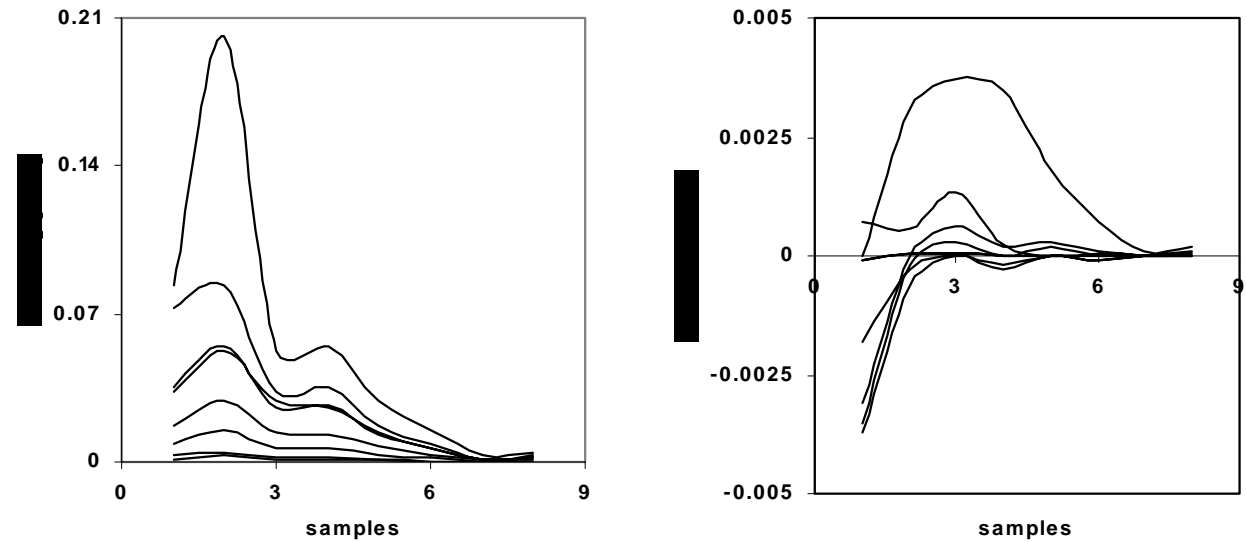

Figure 4. Synchronous (left) and asynchronous (right) 2DCOOR spectra of HS subfractions from a sediment sample 


\subsection{FTIR spectroscopy and 2D correlation analysis in ecotoxicological studies}

The acetone solutions of algal pigments analysed by FTIR spectroscopy show the molecular modifications present in algal cells after pollutant exposure. By a general point of view, most bands of lipids (phospholipids, fatty and ester fatty acids), nucleic acids, proteins and polysaccharides coming from exposed cultures show significant changes of absorption with respect to the same bands present in control samples (Figure 5) (Mecozzi et al., 2007).

However, FTIR spectroscopy allows only a preliminary differentiation among the molecular modifications caused by the action of the examined pollutants, differentiation which can be improved by 2DCORR analysis. Surfactants (both anionic and non ionic) cause variations into cell wall and cytoplasm membrane composition because carbohydrates, lipids, proteins and nucleic acids show changes of concentrations occurring with a significant correlation (i.e bands between 650 and $1750 \mathrm{~cm}^{-1}$ ), while they do not cause modifications of structural (i.e. hydrogen bond) interactions among these biomolecules. In fact, synchronous and asynchronous crosspeaks related to $-\mathrm{OH}$ group (i.e. at $3400 \mathrm{~cm}^{-1}$ ) are absent (Figure 6, middle spectra).

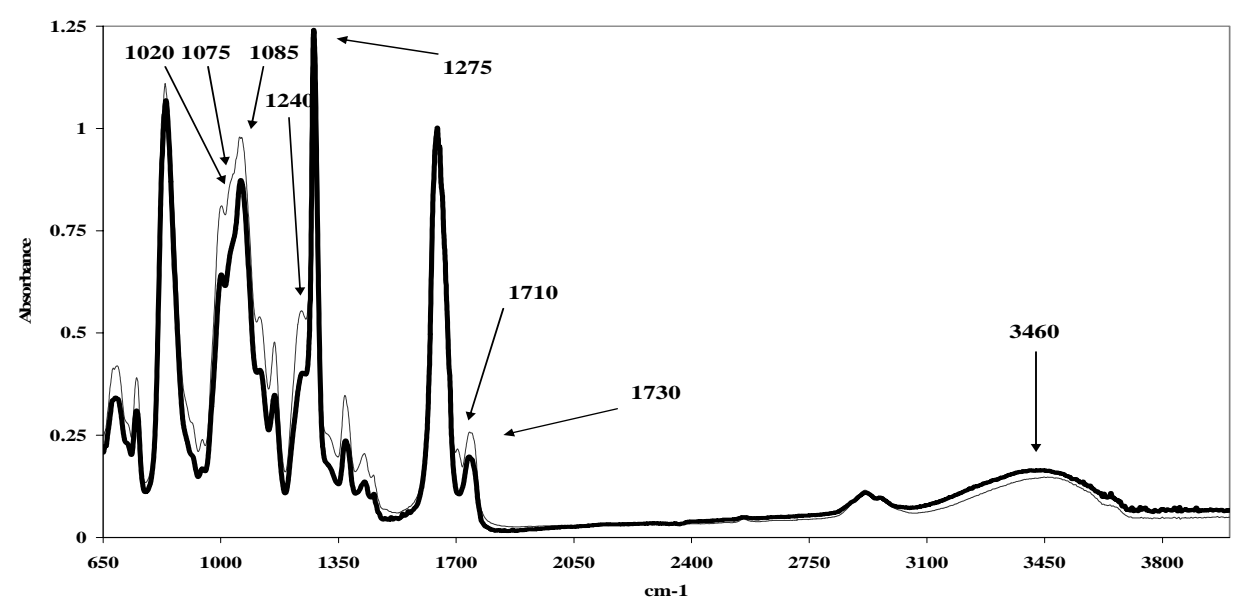

Figure 5. FITR spectra of algal pigment before (grey line) and after exposure (black line) to a $20 \mathrm{mg} \mathrm{L}^{-1} \mathrm{Zn}$ solution

Vice versa, heavy metals such as $\mathrm{Cu}, \mathrm{Hg}$ and $\mathrm{Cr}$ show both variations into cell wall and cytoplasm membrane composition and structural modifications of the interactions among these biomolecules. As shown by Figure 6 (bottom spectra) in addition to several crosspeaks between 650 and $1750 \mathrm{~cm}^{-1}$ related to variation of carbohydrates lipids and proteins, significant crosspeaks related to -OH groups are also present both in synchronous and asynchronous spectra. These findings show that heavy metals have a double toxic mechanism involving quantitative and structural modifications within living cells.

Other polar pollutants such as glycol compounds have an intermediated feature showing relevant variations into cell wall and cytoplasm membrane composition and a weak but not negligible effect of structural modifications among biomolecules (Figure 6, upper spectra). Here, crosspeaks related to $-\mathrm{OH}$ groups are present as weak asynchronous crosspeaks only. This means that glycol compounds produce a weak structural modification within living cells, a modification not linearly correlated with the chemical modifications observed for carbohydrates, proteins and lipids.

2DCORR SS analysis confirmed the general results obtained by 2DCORR WW analysis of FTIR spectra. The similar shape observed within synchronous and asynchronous spectra of algal pigments submitted to all the pollutants included in this study show the natural evolution of the toxic action depending on the increased pollutant concentration (Figure 7). In addition, the observed similarity among the shapes of SS spectra for each set of samples and the absence of some discontinuity points, shows the simultaneous sensibility of cell wall and cytoplasmatic membrane which is a fundamental characteristics of any biomarker of environmental quality (Walsh and Merrill, 1984). 

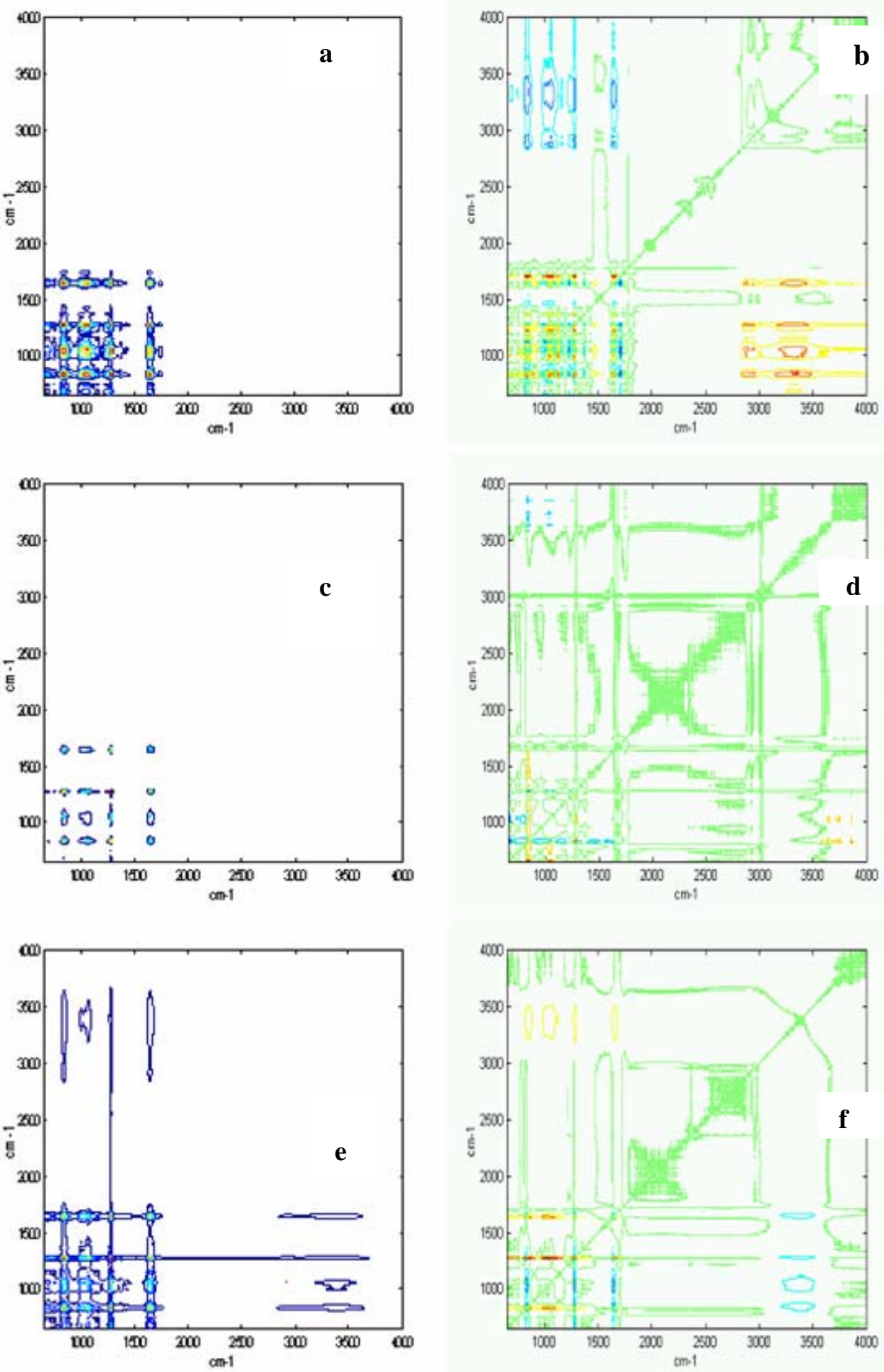

Figure 6. 2DCORR synchronous (left) and asynchronous (right) of algal pigments submitted to diethylen glycol (upper), sodiumlaurilsulphate (middle) and $\mathrm{Hg}$ (bottom) plots 

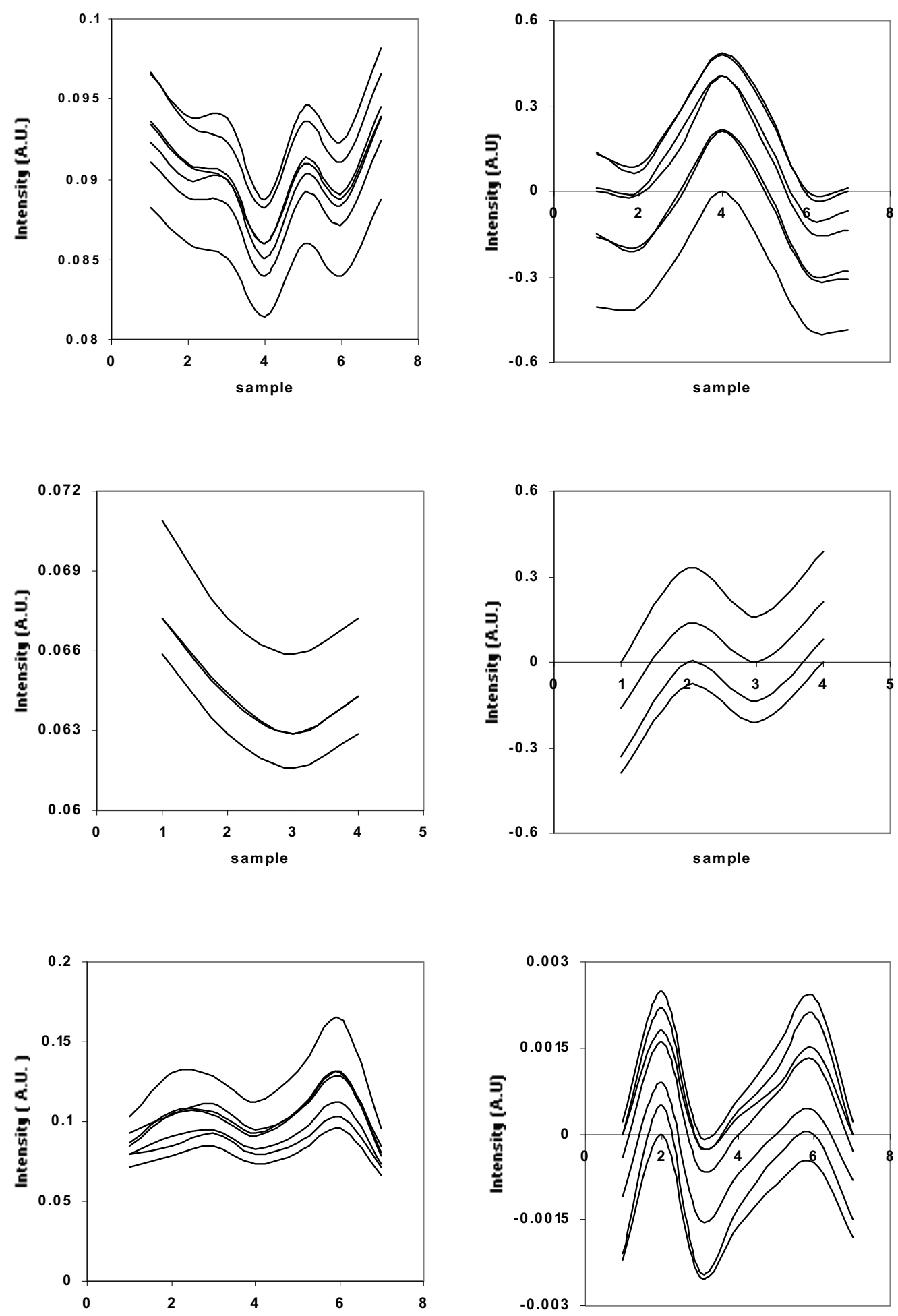

Figure 7. 2DCORR SS spectra for the same samples of Figure 6

\section{CONCLUSION}

The joint application of FTIR spectroscopy and 2DCORR reveals to be a promising technique for detailed environmental studies. In the study of the polymerisation/aggregation of HS from marine sediments FTIR spectroscopy and 2DCORR allow to evidence the different role played by carbohydrates proteins and lipids during the polymerization reaction leading from lower to higher mw aggregates. In the study of the molecular modifications caused by several pollutants in the marine algae Dunaliella tertiolecta, FTIR spectroscopy and 2DCORR allow the differentiation of the toxic effects arising from different pollutants. These findings can be 
helpful tools to identify the most suitable organisms to be used as biomarkers of environmental quality.

\section{REFERENCES}

EPA (1991) Method 600/4-90-027, Methods for measuring the acute toxicity of effluents and receiving waters to freshwater and marine organisms, 4th edition, Cincinnati, USA

Ishiwatari R. (1992) Macromolecular materials (humic substance) in the water column and sediments, Mar. Chem., 39, 151-166

Kögel-Knaber I. (2000) Analytical approaches for characterizing soil organic matter, Org. Geochem., 31, 609-625

Mecozzi M. and Pietrantonio E. (2006) Carbohydrates proteins and lipids in fulvic and humic acids of sediments and its relationships with mucilaginous aggregates in the Italian seas, Mar. Chem., 101, 27-39

Mecozzi M., Pietroletti M and Di-Mento R. (2007) Application of FTIR spectroscopy in ecotoxicological studies supported by Multivariate analysis and 2D correlation spectroscopy, Vibr. Spect., 44, 228-235.

Noda I. and Ozaki I. (2005) Two-dimensional correlation spectroscopy. Application in vibrational and optical spectroscopy, John Wiley \& Sons, UK

Šašic S., Amari T. and Ozaky Y. (2001) Sample-sample and wavenumber-wavenumber twodimensional correlation analysis of attenuated total reflection infrared spectra of polycondensation reaction of bis(hydroxyethylterephthalate), Anal. Chem., 73, 5184-5190

Verdugo P., Alldredge A.L., Azam F., Kirchman D.L., Passow U. and Santschi P.H. (2004) The oceanic gel phase: a bridge in the DOMPOM continuum, Mar. Chem., 92, 67-85.

Walsh G.E. and R.G. Merrill R.G., (1984) Algal bioassays of industrial and energy process effluents, in Algae as ecological indicators, Shubert L.E. (ed.), Academic Press, London. 